\title{
Estimation of exit temperatures in the isentropic compression of real gases
}

\author{
${ }^{1}$ MONAGO, KO; ${ }^{2}$ DAWODU, OT \\ ${ }^{*}$ Department of Pure and Industrial Chemistry, ${ }^{2}$ School of Science Laboratory Technology, University of Port Harcourt, \\ P M B 5323 Choba, Port Harcourt, Nigeria. Email: ken.monago@uniport.edu.ng \\ Tel.: +2348032756950
}

\begin{abstract}
This paper presents the estimation of exit temperatures in the isentropic compression of real gases based on the Peng-Robinson equation of state and entropy balance method. The methods were applied to $\mathrm{Ar}, \mathrm{N}_{2}, \mathrm{CH}_{4}$, $\mathrm{CO}_{2}, \mathrm{C}_{2} \mathrm{H}_{4}$ and $\mathrm{C}_{2} \mathrm{H}_{6}$. Data obtained revealed that isentropic exponent method provides useful results for monatomic and diatomic gases while it is less useful when applied to polyatomic gases. Furthermore, the results obtained in this work show that the use of relative gravity to correlate compressor calculations for gaseous mixtures can lead to serious error in certain cases.
\end{abstract}

DOI: https://dx.doi.org/10.4314/jasem.v22i1.11

Copyright: Copyright (C) 2017 Monago and Dawodu. This is an open access article distributed under the Creative Commons Attribution License (CCL), which permits unrestricted use, distribution, and reproduction in any medium, provided the original work is properly cited

Dates: Received 11 November 2017; received in revised form 15 December 2017; accepted 29 December 2017

Keywords: Peng-Robinson equation of state, isentropic compression, entropy balance, pressure-volume exponent method

Two ideal thermodynamic paths have proved useful in the analyses of processes in which real gases are compressed between fixed pressure limits; these are: reversible adiabatic and polytropic paths.

When a gas is compressed along an adiabatic path between pressure limits which are fixed, the exit temperature is unknown and must be determined from one of two methods; namely,

(a) entropy balance method, or, (b)isentropic exponent methods.

An entropy balance can be accomplished with a Mollier (enthalpy - entropy) chart or, algebraically, by solving an entropy balance equation. However, few Mollier charts are available on the open literature; consequently, to use the entropy balance method, one has to proceed by the algebraic route. The isentropic exponent methods are intuitive extensions of the ideal gas equation to real gases and assume not only that the gas is perfect but, also, that the ratio of the two principal heat capacity functions is independent of temperature.

Today, with the availability of cubic equation of state (EOS) methods that are sufficiently accurate for engineering applications, it should be possible to setup an entropy balance equation from which one may calculate the gas exit temperatures in the isentropic compression of gases. This work is motivated, in part, by the realisation that the petroleum industry in Nigeria still relies on Mollier charts by Brown
(Brown, 1944), which dates back to the 1940s, for solving gas compression problems (Ikoku, 1984). Therefore, the objective of this work is to derive the necessary working equations and to calculate exit temperatures in the isentropic expansion of real gases based on the Peng-Robinson EOS and entropy balance method.

\section{The Equation of State}

The shaft work, $W_{\mathrm{x}}$, done in a flow process is given by (Bett et al., 1982)

$W_{\mathrm{x}}=\int_{1}^{2} V \mathrm{~d} p$

Eq. (1) shows that any EOS method that can predict accurate molar volume, $V$, will, in principle, also lead to good values for the shaft work; in the present case, the work of isentropic compression. The virial EOS can predict the molar volume of gases to better than 0.02 percent accuracy; however, its usefulness is limited to about one-half of the critical density or about $12 \mathrm{MPa}$ in pressure (Monago and Otobrise, 2016; Wiebke et al, 2011). The Lee-Kesler corresponding states method can predict the molar volume of gases and liquids to within an accuracy of 1 percent (Lee and Kesler, 1975); unfortunately, it is mathematically complex and requires considerable computer resources for implementation in process flowsheeting. Although cubic EOS methods, in general, can predict the molar volume of gases and liquids only to within accuracies of about $1-5$ per 
cent they are mathematically simple functions that, relatively speaking, require minimal computer time to implement in process flowsheeting. Among this class of equations, the Peng-Robinson EOS, eq. (2), gives the most accurate values of molar volume (Assael et al, 1996); consequently, it was nominated, from among other cubic EOS methods, for this work.

$$
P=\frac{R T}{V-b}-\frac{a}{\left(V+t_{1} b\right)\left(V+t_{2} b\right)}
$$

In eq. (2), $P$ is pressure, $T$ is thermodynamic temperature, $a$ is an energy parameter, $b$ is a volume parameter and $R$ is the molar gas constant; $t_{1}$ and $t_{2}$ are pure numbers, defined as follows: $t_{1}=1+\sqrt{2}$ and $t_{2}=1-\sqrt{2}$

\section{MATERIALS AND METHODS}

Thermodynamic Analyses

\section{Isentropic compression of real pure gases}

An isentrope of a gas, whether ideal or real, satisfies the differential equation (Monago, 2002)

$$
(\partial V / \partial T)_{\mathrm{p}} \mathrm{d} P+\gamma(\partial P / \partial T)_{\mathrm{v}} \mathrm{d} V=0
$$

In eq. (3), $\gamma=C_{\mathrm{p}} / C_{\mathrm{v}} ; C_{\mathrm{p}}$ is the isobaric heat capacity and $C_{\mathrm{v}}$ is the isochoric analogue. The above equation may be solved in order to calculate temperatures along a line of constant entropy; however, it is computationally easier and more elegant to apply the condition

$$
S_{2}=S_{1}
$$

Where, $S_{\mathrm{i}}$ is the molar entropy at state i. At one time, eq. (4) was implemented by summing entropy changes around a cycle of processes, the proposition being that the sum of entropies around a cyclic process is zero; hence, the procedure became known as the entropy balance method. The molar entropy of a fluid at state $i$, is given by (Monago, 2016).

$$
\begin{aligned}
\frac{S_{\mathrm{i}}(T, P)}{R}= & \frac{S^{\mathrm{pg}}\left(T_{0}, P_{0}\right)}{R}-\ln \left(P_{i} / P_{0}\right)+\frac{1}{R} \int_{T_{0}}^{T_{\mathrm{i}}} \frac{C_{\mathrm{pg}}^{\mathrm{pg}}}{T} \mathrm{~d} T+\ln Z_{\mathrm{i}} \\
& +\frac{1}{R} \int_{\infty}^{V_{\mathrm{i}}}\left[(\partial P / \partial T)_{\mathrm{v}}-R / V\right] \mathrm{d} V
\end{aligned}
$$

In eq. (5), superscript 'pg' indicates the ideal gas state and subscript 0 indicates a reference state.

The compressibility factor at state $\mathrm{i}(P, T)$ is defined by

$$
Z=\frac{p V_{i}}{R T} \text {. }
$$

The heat capacity of the gas in the hypothetical idealgas state, $\boldsymbol{C}_{\boldsymbol{p}}^{\boldsymbol{g}}$, is traditionally given by

$$
C_{\mathrm{p}}^{\mathrm{pg}}=a_{c}+b_{c} T+c_{c} T^{2}+d_{c} T^{3}
$$

Where, $a_{\mathrm{c}}, b_{\mathrm{c}}, c_{\mathrm{c}}$ and $d_{\mathrm{c}}$ are constant coefficients. From eq. (2) and (5), one obtains that if a gas obeys the Peng-Robinson EOS, its entropy at state $i$ is given by:

$$
\begin{aligned}
\frac{S_{\mathrm{i}}(T, P)}{R}= & \frac{S^{\mathrm{pg}}\left(T_{0}, P_{0}\right)}{R}-\ln \left(P_{\mathrm{i}} / P_{0}\right)+\frac{1}{R} \int_{T_{0}}^{T_{\mathrm{i}}} \frac{C_{\mathrm{P}}^{\mathrm{pg}}}{T} \mathrm{~d} T+\ln \left(Z_{\mathrm{i}}-B_{\mathrm{i}}\right) \\
& +\frac{A_{T}^{\prime}}{\left(t_{1}-t_{2}\right) B_{\mathrm{i}}} \ln \left(\frac{Z_{\mathrm{i}}+t_{1} B_{\mathrm{i}}}{Z_{\mathrm{i}}+t_{2} B_{\mathrm{i}}}\right)
\end{aligned}
$$

Where, $B=(P b / R T)$ is dimensionless volume parameter; $A=a P /(R T)^{2}$ is dimensionless energy parameter. From eq. (4) and (7), one obtains a computational equation in terms of $T_{2}$ that is satisfied along an isentrope of a real gas; within the framework of the Peng-Robinson EOS

$$
\begin{aligned}
F(T) & =\frac{1}{R}\left\{a_{c} \ln \left(T_{2} / T_{1}\right)+b_{c}\left(T_{2}-T_{1}\right)+\frac{C_{c}\left(T_{2}^{2}-T_{1}^{2}\right)}{2}+\frac{d_{c}\left(T_{2}^{3}-T_{1}^{3}\right.}{3}\right\}+ \\
& +\ln \frac{Z_{2}-B_{2}}{Z_{1}-B_{1}}+\frac{A_{T, 2}^{\prime}}{\left(t_{1}-t_{2}\right) B_{2}} \ln \left(\frac{Z_{2}+t_{1} B_{2}}{Z_{2}+t_{2} B_{2}}\right)-\frac{A_{T, 1}^{\prime}}{\left(t_{1}-t_{2}\right) B_{2}} \ln \left(\frac{Z_{1}+t_{1} B_{1}}{Z_{1}+t_{2} B_{1}}\right)-\ln \left(P_{2} / P_{1}\right)
\end{aligned}
$$

Where, $A_{T}^{\prime}=a_{T}^{\prime} P /(R T)^{2}$ is dimensionless first temperature derivative of the energy parameter, and $a_{T}^{\prime}=T \frac{\mathrm{d} a}{\mathrm{~d} T}=-m a_{c} \sqrt{\alpha T_{r}}$

$T_{\mathrm{r}}$ is reduced temperature; $T_{\mathrm{r}}=T / T_{\mathrm{c}}$, and $T_{\mathrm{c}}$ is critical temperature.

\section{Reversible isothermal compression of real pure gases}

The usefulness of the reversible isothermal path lies in its position as a limit process: minimum work of compression is expended along a reversible isothermal path. For a fluid that obeys the PengRobinson EOS, its consequences are readily set out $W_{x}^{i s o}=R T \ln \left(Z_{2} / Z_{1}\right)-R T \ln \left(P_{2} / P_{1}\right)-R T\left(Z_{2}-Z_{1}\right)$

$$
\begin{aligned}
& H_{2}^{\text {so }}-H_{1}^{\text {so }}=\Delta H^{\text {so }} \\
& =R\left(Z_{2}-Z_{1}\right)+\frac{A_{T, 2}^{\prime}-A_{2}}{\left(t_{1}-t_{2}\right) B_{2}} \ln \frac{Z_{2}+t_{1} B_{2}}{Z_{2}+t_{2} B_{2}} \frac{A_{r, 1}-A_{1}}{\left(t_{1}-t_{2}\right) B} \ln \frac{Z_{1}+t_{1} B}{Z_{1}+t_{2} B}
\end{aligned}
$$

And, following the engineering sign convention for $W_{\mathrm{x}}$,

$$
Q_{\text {iso }}=W_{x}^{\text {iso }}+\Delta H^{i s o}
$$

In eq. (10) - (12), $H_{i}^{\text {iso }}$ is the enthalpy of the gas at state $i, W_{x}^{\text {iso }}$ is the work of compression and $Q_{\text {iso }}$ is the heat transfer along the isothermal path. 


\section{Compression of mixtures:}

For mixtures, some of the quantities defined in section 3.1 to 3.2 need to be modified according to the following prescriptions. The energy parameter for a mixture, $a_{\text {mix }}$, is given by the van der Waals onefluid mixing rule:

$$
a_{\text {mix }}=\sum_{\mathrm{i}=1}^{c} \sum_{\mathrm{j}=1}^{c} x_{\mathrm{i}} x_{\mathrm{j}}\left(a_{\mathrm{i}} a_{\mathrm{j}}\right)^{1 / 2}
$$

The volume parameter for a mixture, $b_{\text {mix }}$, is taken as a simple mole-fraction-weighted average of the pure component values:

$$
b_{\text {mix }}=\sum_{\mathrm{i}=1}^{c} x_{\mathrm{i}} b_{\mathrm{i}}
$$

Where, in eq. (13) and (14) $a_{\mathrm{i}}$ and $b_{\mathrm{i}}$ are, respectively, the energy and volume parameters of pure i. The dimensionless analogues of $A_{\text {mix }}$ and $B_{\text {mix }}$ for mixtures are similarly defined:

$$
\begin{aligned}
& A_{\text {mix }}=\sum_{\mathrm{i}=1}^{c} \sum_{\mathrm{j}=1}^{c} x_{\mathrm{i}} x_{\mathrm{j}}\left(A_{\mathrm{i}} A_{\mathrm{j}}\right)^{1 / 2} \\
& B_{\text {mix }}=\sum_{\mathrm{i}=1}^{c} x_{\mathrm{i}} B_{\mathrm{i}}
\end{aligned}
$$

\section{Derived Thermodynamic Properties:}

Once eq. (8) is solved for the exit temperature, $T_{2}$; other thermodynamic properties can be calculated within the framework of the particular equation of state, which here is the Peng-Robinson equation. The enthalpy at state 2 is given by

$$
\begin{aligned}
H_{2}(T, P) & =\frac{H^{g}\left(T_{0}\right)}{R T}+\frac{1}{R T}\left\{a_{c}\left(T-T_{0}\right)+\frac{b_{c}\left(T^{2}-T_{0}^{2}\right)}{2}+\frac{C_{c}\left(T^{3}-T_{0}^{3}\right)}{3}+\frac{d_{c}\left(T^{4}-T_{0}^{4}\right)}{4}\right\} \\
& +Z-1+\frac{A_{T}-A}{\left(t_{1}-t_{2}\right) B} \ln \frac{Z+t_{1} B}{Z+t_{2} B}
\end{aligned}
$$

The work done in the isentropic compression is given by

$$
W_{\mathrm{x}}=-\left(H_{2}-H_{1}\right)
$$

\section{Numerical Methods:}

There can be up to three roots for $V(T, P)$ of eq. (2); the largest of which corresponds to the gas phase, the smallest to the liquid phase, and the intermediate one (which corresponds to a mechanically-unstable state of negative compressibility) has no physical significance. The largest root is typically about three to four orders of magnitude larger than the smallest one such that in computational work solving for $V(T, P)$ makes the problem an ill-conditioned calculation. However, substituting eq. (6) into eq. (2) and simplifying, gives eq. (19).

$$
Z^{3}-(1-B) Z^{2}+\left(A-2 B-3 B^{2}\right) Z-\left(A B-B^{2}-B^{3}\right)=0
$$

Eq. (19), like eq. (2), can possess up to three roots in $Z(T, P)$, which correspond to those of $V(T, P)$; however, with respect to $Z(T, P)$, the difference between the largest and smallest roots is at most one order of magnitude and computationally leads to a well-posed problem. Therefore, we first solved eq. (19) and (8), successively, by iteration until convergence is achieved in eq. (8); this gives the exit, or final, temperature, $T_{2}$. Eq. (19) was solved by the so-called modified Richmond method (Edmister and Lee, 1984) and in solving that equation for $Z$, iteration was stopped when $F(Z) \leq 10^{-6}$. Equation (8) was solved by the Newton-Raphson method (Faires and Burden, 1993) and iteration was terminated when $F(T) \leq 10^{-5}$.

A number of calculations were performed in order to validate the accuracy of the expressions derived in this work and their computational implementation. All algebraic expressions derived here for derivative properties were compared with values obtained from numerical differentiation, using the five-point midpoint formula. For the second derivatives properties, a three-point mid-point formula was used instead. Typically, values obtained from numerical differentiation agreed with the algebraic analogue up to the 4 th or 5 th decimal place. We calculated compressibility factors for methane along a number of isotherms using sections of the program code employed in this work and the results were compared with the data of Monago (2007); agreement of $1-3$ $\%$ errors expected of a cubic equation were obtained. Finally, for reversible adiabatic compression, exit temperatures obtained from eq. (8) were used to calculate the entropy change for the process 17 ) concerned and in all the cases that we investigated, the condition $\Delta S=0$ was satisfied to the 5 th or 6 th decimal place.

\section{RESULTS AND DISCUSSION}

The results obtained were correlated in terms of the temperature - pressure exponent method; namely,

$T_{2} / T_{1}=\left(P_{2} / P_{1}\right)^{m}$

Two assumptions were made to derive eq. (20); namely, that the gas is ideal, and that the ratio of the two principal heat capacity functions, $\gamma=C_{\mathrm{p}} / C_{\mathrm{v}}$, is a constant, independent of temperature. For an ideal gas, $\gamma$ and $m$ are related by: $\gamma^{\mathrm{pg}}=1 /(1-m)$. 


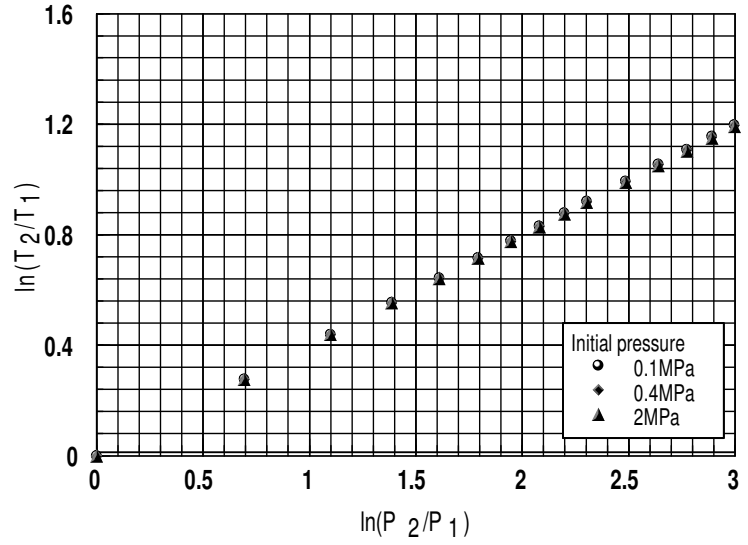

Fig. 1: Calculated isentropic temperature - pressure diagram of argon

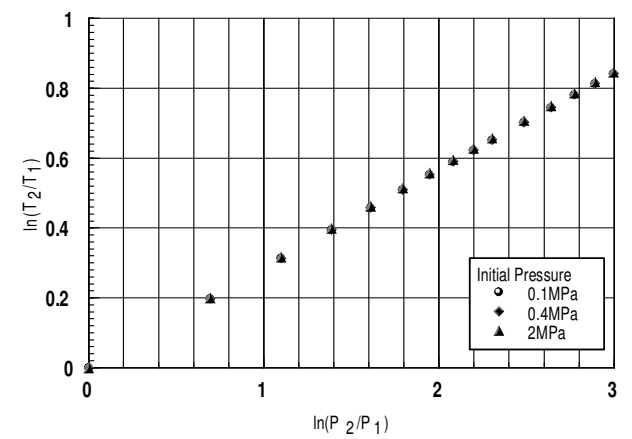

Fig 2: Calculated isentropic temperature - pressure diagram for $\mathrm{N}_{2}$

Monatomic gases: The second of the two assumptions made above with respect to eq. (20) is unassailable for monatomic gases of which argon is prototypical. Fig 1 shows that for argon, the isentropic temperature - pressure exponent is, to a high degree of accuracy, linear and the slope is a constant, independent of temperature and density, in accordance with eq. (20). Furthermore, Fig. 1 predicts a value of $\gamma=1 /(1-m)$ that equals its theoretical value of 1.67; see also table 2 . Therefore, one may reasonably conclude that in gas compression, it is the temperature dependence of the heat capacity ratio that determines the exponent and that residual contribution to properties plays a relatively minor role.
Diatomic gases: For gases composed of rigid molecules, provided that the quotient $T / \Theta^{\text {ot }}$ (Where $\Theta^{\text {ot }}$ is the rotational characteristic temperature) is sufficiently high for the classical approximation to be valid, the variation of $\gamma$ with temperature arises solely from the vibrational contribution to the partition function. A rigid diatomic molecule has one vibrational mode; for $\mathrm{N}_{2}$, the vibrational characteristic temperature, $\Theta^{\mathrm{vib}}=3340 \mathrm{~K}$; therefore, the vibrational contribution to heat capacity may be given by:

$C_{\mathrm{p}, \mathrm{vib}}-C_{\mathrm{v}, \mathrm{vib}}=R\left(1-2 \Theta^{\mathrm{vib}} / T\right)$

One may see from eq. (21) that for a typical diatomic molecule, the assumption that $\gamma$ is independent of temperature is a good one, provided that temperature is not greater than about $1000 \mathrm{~K} . \mathrm{N}_{2}$ is the prototypical linear diatomic molecule and its isentropic temperature - pressure diagram is displayed in Fig. 2 from which it is seen that the present results follow eq. (20) with good accuracy.

Polyatomic gases: $\mathrm{CO}_{2}$ has four vibrational modes, of which two are degenerate, the vibrational characteristic temperatures are: 960 K, 960 K, 1998 $\mathrm{K}$ and $3380 \mathrm{~K}$; for this molecule, the vibrational contribution to heat capacity may be approximated by the expression

$C_{\mathrm{p}, \mathrm{vib}}-C_{\mathrm{v}, \mathrm{vib}}=R \sum_{i=1}^{4}\left\{1-2 x_{i}+\left(3 x_{i} / 2\right)^{2}\right\}$

Where, $x_{\mathrm{i}}=\Theta^{\mathrm{vib}, \mathrm{i}} / T$. From eq. (22) we see that the vibrational contribution to heat capacity is a function of temperature. Fig. 3 shows the isentropic temperature - pressure diagram for $\mathrm{CO}_{2}$ from which it is seen that whereas the exponent remains reasonably linear, it is not a constant. Other polyatomic gases investigated were $\mathrm{CH}_{4}, \mathrm{C}_{2} \mathrm{H}_{4}$ and $\mathrm{C}_{2} \mathrm{H}_{6}$.

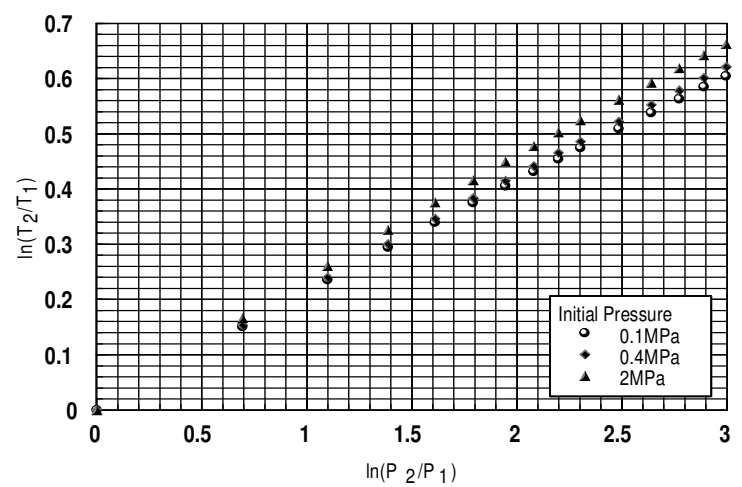

Fig 3: Isentropic temperature - pressure diagram for $\mathrm{CO}_{2}$. 
Mixtures: The petroleum industry in Nigeria uses 'apparent molecular weight' of a gas mixture as a parameter to perform compressor calculations (Ikoku, 1984). The proposition is that if two mixtures have the same value for this parameter, they will also have the same compression characteristics. To test this hypothesis, we have constructed two natural gas mixtures, which have the same apparent molecular weight, but have different compositions; table 1 . The mixtures are identified as mixture I and II.

Table 1: Two natural gas with different compositions, but identical apparent molecular weight

\begin{tabular}{lcc}
\hline Component & \multicolumn{2}{c}{ Mole fraction } \\
\cline { 2 - 3 } & Mixture & Mixture II \\
\hline $\mathrm{CH}_{4}$ & 0.83 & 0.78 \\
$\mathrm{C}_{2} \mathrm{H}_{6}$ & 0.07 & 0.05 \\
$\mathrm{C}_{3} \mathrm{H}_{8}$ & 0.05 & 0.03 \\
$\mathrm{n} \mathrm{C}_{4} \mathrm{H}_{10}$ & 0.05 & 0.02 \\
$\mathrm{H}_{2} \mathrm{~S}$ & 0 & 0.03 \\
$\mathrm{~N}_{2}$ & 0 & 0.06 \\
$\mathrm{CO}_{2}$ & 0 & 0.03 \\
\hline
\end{tabular}

According to the above hypothesis, mixture I and II should have the same isentropic temperature pressure exponent; however, as table 2 shows, the mixtures gave different values for the exponents $m$ and $\gamma^{\text {ig. }}$. Furthermore, Fig. 4 and 5 show that the mixtures have different isentropic temperature pressure exponent diagrams.

Table 2: Comparison of theoretical and calculated isentropic temperature - pressure exponents $\gamma^{\text {theor }}=$ theoretical value of $\gamma, \gamma^{\text {alc }}=$ value of $\gamma$ obtained in this work.

\begin{tabular}{llll}
\hline Substance & $M$ & $\gamma^{\text {theor }}$ & \multicolumn{1}{c}{$\gamma^{\text {calc }}$} \\
\hline $\mathrm{Ar}$ & 0.4 & 1.67 & 1.67 \\
$\mathrm{~N}_{2}$ & 0.27 & 1.40 & 1.37 \\
$\mathrm{CO}_{2}$ & 0.2 & 1.20 & 1.25 \\
$\mathrm{CH} 4$ & $0.2 \pm 0.05$ & 1.20 & $1.26 \pm 0.01$ \\
$\mathrm{C}_{2} \mathrm{H}_{4}$ & $0.17 \pm 0.1$ & 1.20 & $1.205 \pm 0.015$ \\
$\mathrm{C}_{2} \mathrm{H}_{6}$ & $0.14 \pm 0.1$ & 1.20 & $1.165 \pm 0.015$ \\
\hline
\end{tabular}

Table 2 summarizes the results obtained in this work for the isentropic temperature - pressure exponents of some gaseous substances, while fig. 6 plots the differences between exit temperatures in the compression of mixture I and II as functions of temperature.

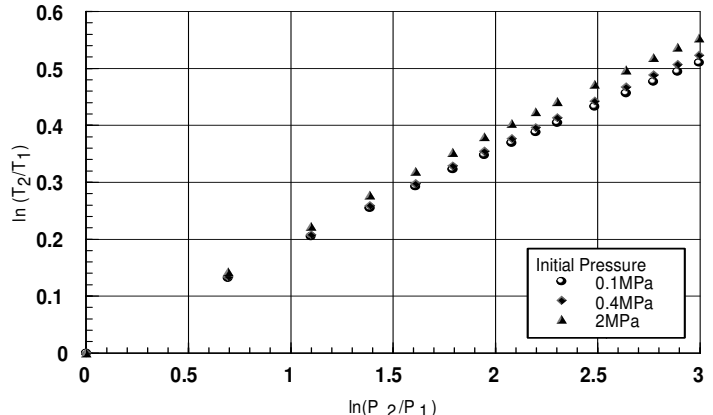

Fig 4: Temperature - pressure diagram for mixture I

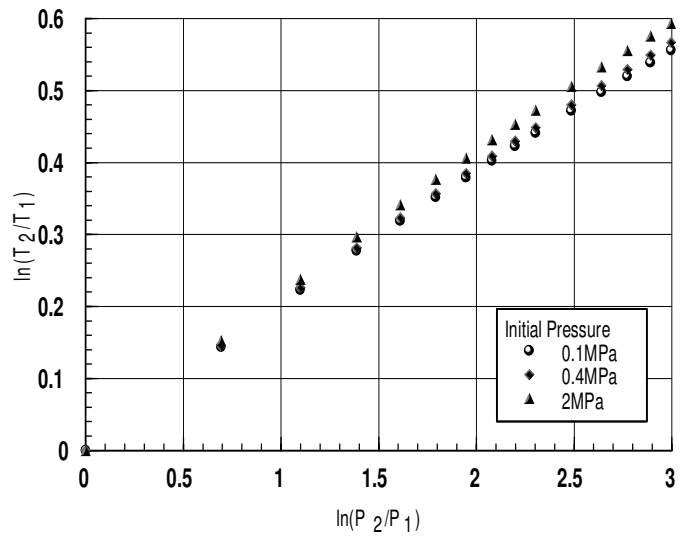

Fig 5: Temperature - pressure diagram for mixture II

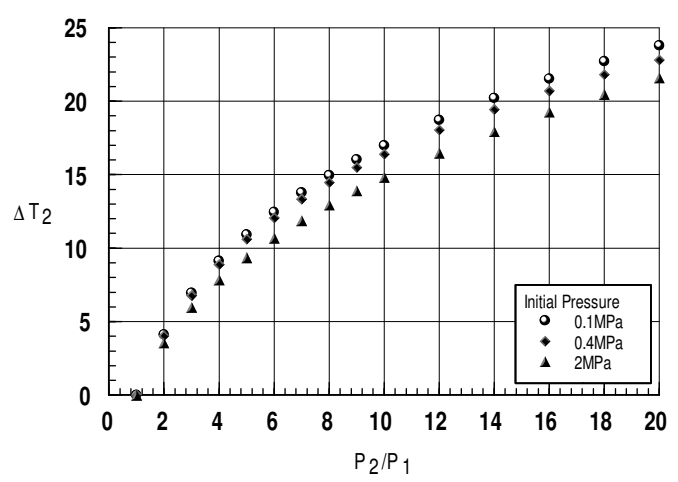

Fig 6: Deviations in exit the temperatures of mixture I and II as a function of compression ratio.

\section{Conclusion:}

Using the Peng-Robinson EOS and the entropy balance method, we derived a computational expression for exit temperatures in the reversible isentropic compression of real gases between fixed pressure limits. Calculations were performed to determine $T_{2}$ and $W_{\mathrm{x}}$ for the following gases: Ar, $\mathrm{N}_{2}$, $\mathrm{CH}_{4}, \mathrm{CO}_{2}, \mathrm{C}_{2} \mathrm{H}_{4}$ and $\mathrm{C}_{2} \mathrm{H}_{6}$. It was shown that, while the temperature - pressure exponent method gave useful results for monatomic and diatomic gases, it 
was rather approximate for polyatomic gases because the exponent turned out not to be a constant for the latter set of gases. Further, the results obtained in this work show that the use of apparent molecular weight to correlate compressor calculations for gaseous mixtures, while it works well for natural gas composed entirely of hydrocarbons, it may lead to significant error if applied to natural gas that contains inorganic gases.

\section{REFERENCES}

Assael M.; Trusler J. P. M; Tsolakis T F (1996). Thermophysical Properties of Fluids, Imperial College Press (Lond), p. $111-160$.

Bett K E; Rowlinson J S; Saville G (1982). Thermodynamics for Chemical Engineers, (1982), Athlone Press (Lond), p. 23; p 197

Brown G. G. (1944). Mollier charts for natural gas systems Trans AIMME, July 1944, Tech Paper no 1747 .

Edmister W. C.; Lee B I (1984). Applied Hydrocarbon Thermodynamics, Gulf Publishing Co (Houston), p. 226.

Faires J D; Burden, R L (1993). Numerical Methods, PWS Publishing Co (Boston), p 37
Ikoku C. (1984). Natural gas production engineering, John Wiley (New York), p. 95 - 99.

Lee, B. I.; Kesler, M G (1975). A generalized thermodynamic correlation based on threeparameter corresponding states, AIChE J., 21, $510-519$.

Monago, K O (2002). Selected topic on applied physical chemistry, Tinop (Nigeria), p.74

Monago, K O (2007). An extended equation for gaseous methane determined from the speed of sound, Chem. Phys., 337, 125 - 134.

Monago, K. O. (2016). Tables of thermodynamic properties, $2^{\text {nd }}$ ed., Splendid (Lagos), p. 34.

Monago, K O; Otobrise, C (2016). Virial coefficients of nitrogen from a quadrupolar site-site potential function, J. Comp. Theor. Chem., 11, 38-47.

Wiebke, J.; Scherdtfeger, P; Moyano, G.; Pahl, E (2011). An atomistic fourth-order virial equation of state for argon from first principles calculations, Chem. Phys. Lett., 514, 164 - 187. 